\title{
SOME CONSEQUENCES OF LEFT INVERTIBILITY
}

\author{
SCOTT H. HOCHWALD AND BERNARD B. MORREL
}

\begin{abstract}
Let $A$ be a noncommutative Banach algebra with identity $e$. Let $L$ be a multiplicative semigroup of left-invertible elements of $A$ which properly contains the invertible elements of $A$. Then there does not exist a function $g: L \rightarrow A$ such that $g(a b)=g(b) g(a)$ and $g(a) a=e$ for all elements $a$ and $b$ of $L$. This paper contains an elementary proof of this result, and thereby answers a question posed by G. R. Allan.
\end{abstract}

1. Introduction. Let $A$ be a noncommutative Banach algebra with identity $e$ and norm $\|\cdot\|$. If $a$ and $b$ are invertible elements of $A$, then $(a b)^{-1}=b^{-1} a^{-1}$ and $a^{-1} a=e$. This is equivalent to saying that there exists a function $g$ on the invertible elements of $A$ such that $g(a b)=g(b) g(a)$ and $g(a) a=e$. Can the domain of the function $g$ be enlarged? In other words, is there a subset $L$ of $A$, necessarily a multiplicative semigroup, which properly contains the set of invertible elements and a function $g$ on $L$ so that $g(a b)=g(b) g(a)$ and $g(a) a=e$ for all $a, b \in L$ ? The main result of this paper is that the answer to this question is no.

A theorem due to G. R. Allan [1, pp. 10-11] handles the case in which $L$ is the set of all left-invertible elements of $A$ and $A$ is complex. He used techniques from the theory of functions of several complex variables to prove his result. He proposed that someone find an elementary proof of this fact for a particular Banach algebra. This last statement needs some elaboration and amplification.

Allan's result actually treats the more general case (in one sense) in which the function in question is holomorphic. It is easy to see that the function $g$ (described above) is holomorphic (if $A$ is complex) on the multiplicative semigroup of leftinvertible elements of $A$. The details are as follows. Let $a_{0}$ be a left-invertible element of $A$, let $g\left(a_{0}\right)=b_{0}$, and let $a$ be any left-invertible element of $A$ with the property that $\left\|a_{0}-a\right\|<1 /\left\|b_{0}\right\|$. Then $a=a-a_{0}+a_{0}=\left[\left(a-a_{0}\right) b_{0}+e\right] a_{0}$. This shows that $a=\left[e-\left(a_{0}-a\right) b_{0}\right] a_{0}$. The element $e-\left(a_{0}-a\right) b_{0}$ is invertible because $\left\|\left(a_{0}-a\right) b_{0}\right\|<1$ (by the restriction on the norm of $\left.a_{0}-a\right)$. In fact, if $v=e-\left(a_{0}-a\right) b_{0}$ then $v^{-1}=\sum_{n=0}^{\infty}\left[\left(a_{0}-a\right) b_{0}\right]^{n}$. Since $g(v) v=e$, it follows that $g(v)=v^{-1}$. Thus,

$$
g(a)=g\left(v a_{0}\right)=b_{0} v^{-1}=b_{0}\left[e+\left(a_{0}-a\right) b_{0}+\left(\left(a_{0}-a\right) b_{0}\right)^{2} v^{-1}\right] .
$$

Therefore,

$$
g(a)=g\left(a_{0}\right)+g^{\prime}\left(a_{0}\right) h+\varepsilon\left(a_{0} ; h\right),
$$

where $h=a-a_{0}, g^{\prime}\left(a_{0}\right) h=-b_{0} h b_{0}$ and

$$
\left\|\varepsilon\left(a_{0} ; h\right)\right\|=\left\|b_{0}\left(\left(a_{0}-a\right) b_{0}\right)^{2} v^{-1}\right\| \leq\|h\|^{2}\left\|b_{0}\right\|^{3}\left\|v^{-1}\right\| .
$$

This shows that $g$ is holomorphic.

Received by the editors March 3, 1986.

1980 Mathematics Subject Classification (1985 Revision). Primary 46H05, 39B70; Secondary 47D05. 
It was mentioned above that Allan chose a particular Banach algebra for his question. The Banach algebra that he chose was $B(H)$, the algebra of bounded linear transformations (operators) on a separable, complex, infinite-dimensional Hilbert space $H$. Obviously, the main result of this paper covers this special case.

The authors wish to thank Donald Sarason for communicating G. R. Allan's question to them.

2. An answer to G. R. Allan's question. An immediate consequence of the following theorem is the nonexistence of the function $g$ described in the first paragraph of $\S 1$.

THEOREM 2.1. Let $A$ be a noncommutative Banach algebra with identity $e$ and norm $\|\cdot\|$. Let $L$ be a multiplicative semigroup of left-invertible elements of $A$ which properly contains the invertible elements of $A$. Then there does not exist a function $g: L \rightarrow A$ such that

(1) $g(a) a=e$ for all $a \in L$,

(2) $g(a b)=g(b) g(a)$ whenever $a, b \in L$ commute and at least one of them is invertible.

ProOF. Assume that $g$ exists and let $a$ be a noninvertible element of $L$. Choose a real number $\lambda$ so that $\lambda>\|a\|$. Then $\lambda e-a$ is invertible. In fact,

$$
(\lambda e-a)^{-1}=\frac{1}{\lambda} \cdot \sum_{n=0}^{\infty}\left(\frac{a}{\lambda}\right)^{n} .
$$

Since $(\lambda e-a)^{-1}(\lambda e-a)=e=(\lambda e-a)(\lambda e-a)^{-1}$, it follows that $(\lambda e-a)^{-1}$ commutes with $a$. This implies that $g\left(a(\lambda e-a)^{-1}\right)=g\left((\lambda e-a)^{-1} a\right)$. It then follows from property (2) that $g\left((\lambda e-a)^{-1}\right) g(a)=g(a) g\left((\lambda e-a)^{-1}\right)$. It then follows from property (1) that $(\lambda e-a) g(a)=g(a)(\lambda e-a)$. This last equation is equivalent to $a g(a)=g(a) a$. Then applying property (1) again gives $g(a) a=e=a g(a)$, a contradiction, since $a$ was assumed to be nonivertible. This completes the proof of this theorem.

At this point it seems appropriate to ask the following nebulous question: Does there exist a function $g: L \rightarrow A$ such that

(1) $g(a) a=e$ for all $a \in L$.

(2) Another condition. ? (Provide an elementary proof.)

( $A, L$, and $e$ are described in Theorem 2.1.)

Possible conditions for (2) are: $g$ is holomorphic, $g$ is continuous or $g\left(a^{2}\right)=(g(a))^{2}$ for all $a \in L$. Allan's results in [1, pp. 10-11] show that $g$ does (not) exist if it is assumed that $g$ is continuous (holomorphic) and $L$ is the multiplicative semigroup of all left-invertible elements of $A$.

Perhaps this question is easier to answer when $A=B(H)$.

\section{REFERENCES}

1. G. R. Allan, Holomorphic left-inverse functions, Proc. Conf. on Banach Algebras and Several Complex Variables (F. Greenleaf and D. Gulick, editors), Contemporary Mathematics, Vol. 32, Amer. Math. Soc., Providence, R.I., 1984.

Department of Mathematical SCIENCES, University of NORTh Florida, JaCKSONVILLE, FLORIDA 32216

I.U.P.U.I., 1201 E. 38TH STREET, INDIANAPOLIS, INDIANA 46205 